\title{
Avena sativa: An Effective Natural Ingredient in Herbal Shampoos for the Treatment of Hair Greasiness
}

\author{
Effat Sadat Farboud ${ }^{1 *}$, Gholamreza Amin ${ }^{2}$ and Leyla Akbari ${ }^{1}$ \\ ${ }^{1}$ Department of Pharmaceutics, School of Pharmacy, Tehran University of Medical \\ Sciences, Tehran, Iran. \\ ${ }^{2}$ Department of Pharmacognosy, School of Pharmacy, Tehran University of Medical \\ Sciences, Tehran, Iran.
}

Authors' contributions

This work was carried out in collaboration between all authors. Author ESF designed the study, performed the statistical analysis, wrote the protocol, and wrote the manuscript. Author GA supervised herbal collection and analysis and author LA managed the literature search and participated in analysis of the study. All authors read and approved the final manuscript.

Research Article

Received $18^{\text {th }}$ July 2012

Accepted $1^{\text {st }}$ December 2012 Published $13^{\text {th }}$ February 2013

\section{ABSTRACT}

Aims: Evaluation of anti-greasiness properties of oat extract and oil in shampoos. Methodology: Seborrhea can cause greasiness in scalp and hair. There are few compounds currently in use to reduce sebaceous gland secretions. Avena sativa or oat is a plant containing saponins as active ingredients with cleansing activity. In a double blinded, randomized, placebo clinical study, we investigated anti-grease and cleansing effects of oat by comparing shampoos formulated from oat extract and oil with control shampoo. Sixty male and female volunteers aged 19 to 30 years old randomly received either oat oil/extract or placebo to the scalp for 5 minutes, twice per week for four weeks. Signs and symptoms such as scaling, greasiness and itching were assessed every week.

Results:In vitro detergency test revealed better detergency power in extract and 
oil of oat shampoos compared with control shampoo. Clinical studies showed a significant reduction of greasiness scores in extract shampoo compared to the oil and control shampoos.

Conclusion: shampoos containing oat extract could be considered as an appropriate alternative therapy for the management of greasiness in seborrheic patients.

Keywords: Avena sativa; oat; shampoo; hair greasiness; herbal; formulation.

\section{INTRODUCTION}

The sensation of "oiliness" or "greasiness" of skin, scalp and hair is very undesirable feeling for most people. To some extent, the perception of "oiliness" or "greasiness" is very personal and may or may not be objectively identified with excess skin oil (sebum) production. A feeling of oiliness in hair may be associated with accumulation or degradation of hair cosmetic products, or by-products of heavy scalp perspiration.

Seborrhea can cause feeling of oily/greasy scalp and hair. Unlike seborrheic dermatitis, however, the oiliness is not associated with crusting, inflammation and intense itching. To our knowledge, no locally applied product can cure acute seborrhoea, and only relatively toxic compounds with serious adverse effects are able to reduce sebaceous gland secretion when given systemically. Due to side effects of systemic routes, topical products such as sulphur and sulphur derivatives, tars, grease absorbers and plant extracts are preferred therapeutic choices [1]. Since ancient times, plants and natural products have been considered as sources for the cosmetic and toiletries. Herbal and natural preparations are currently popular and widely used. Oat (Avena sativa) has been used in traditional and herbal medicine to treat nervous exhaustion, insomnia, weakness of nerves and also as a bath for eczema. Oat is a valuable source of useful proteins and lipids for the skin and hair. The precise center of origin of Avena sativa is not known, but it is generally considered to be originated from Southern Europe and Western Asia. The oat seed contains high amount of soluble silica, iron, manganese and zinc. In addition, it is enriched with triterpene saponins (Avenacin and Avenocoside), scopletin and simple indole alkaloids. Seeds also contain minerals, steroidal compounds, vitamin B1, vitamin B2, vitamin D, vitamin E, carotene [2], lipids and polyphenols [3,4].

Oat has antioxidant property and can reduce free radicals [5]. The high amount of starches and beta-glucan in oat is responsible for its protective and water-holding function which exhibits high viscosity at relatively low concentrations and hence can be used as a thickening agent in pharmacy practices [6]. Moreover, oat lipids especially digalactosyldiacylglycerol form a lamellar liquid crystalline phase with water which makes it an interesting raw material for the cosmetics and pharmaceutical industries [7].

Oatmeal with great anti-irritant and anti-inflammatory properties has been used 
extensively in cosmetic preparations $[8,9]$ to relieve itch and irritation associated with various xerotic dermatoses. Extracts of oats have been shown to inhibit prostaglandin biosynthesis [10] and avenanthramides present in oat are potent anti-inflammatory agents exerting anti irritant effects[11-13]. Vie and coworkers [14] demonstrated that topical application of oat extracts significantly decreased inflammation induced by sodium lauryl sulphate on the volar forearm of human subjects. Matheson reported that a group using the product made of colloidal oatmeal had significantly less itch than those using oil containing liquid paraffin [15]. Anti-inflammatory effect of oatmeal in treatment of rosacea has been also reported [16].Oat is a topical emollient and a colloidal fraction has been successfully used in bath preparations for eczema or dry skin. Oat extract has a moisturizing and anti-aging effect perhaps through its different types of phenolic compounds such as avenanthramides. Compared to other phenolics such as vanillin and caffeic acid, avenantheramides have 10 to 30 times more antioxidant activity $[9,11]$. Oat colloidal extract has also reported to exert healing effects [17]. Some of Avena preparations and products are marketed under different brand names [18].

The cleansing activity of oat is mostly attributed to its saponines. Oatmeal is an effective cleanser which contains saponins to absorb dirt, oil and sebaceous secretions whereas its hydrophilic and lipophilic components absorb and solubilize aqueous debris. Lipids such as oleic acid, linoleic acid, linolenic acid and saturated fatty acids present in oat are suitable for saponification and cleansing activity [19].

In our study we took advantage of the cleansing effect of oat and used extract and oil of the oat in shampoo formulations and compared their anti-grease activities with control regular shampoo.

\section{MATERIALS AND METHODS}

\subsection{Materials}

Texapon N70 (Sodium laureth sulphate 70\%), Texapon SB3 (Sodium Laureth sulfosuccinate 40\%, Dehyton K (Cocamidopropyl betaine 30\%), Comperlan KD (Cocamide DEA, 90\%) were purchased from Henkle, Germany. All other chemicals and solvents were analytical grades.

\subsection{Collection of Avena sativa}

Avena sativa (L. Geraminae) was collected in the beginning of summer from Damavand area, Iran. A voucher specimen has been deposited in the herbarium of School of Pharmacy, Tehran University of Medical Sciences, Tehran, Iran.

\subsection{Preparation of Oil and Total Extract}

The groats were isolated and dried at $40^{\circ} \mathrm{C}$ for seven days and were eventually powdered. Extraction was performed by soxhlet apparatus using a hydroalcoholic 
solvent. The extract was filtered and concentrated. A highly non polar solvent such as hexane was used to extract oil from oat. Physicochemical tests such as color, $\mathrm{pH}$, specific gravity, refractive index, saponification and iodine index were performed for oil extraction. Saponins were extracted from the oat flour and oat extract by using $70 \%$ ethanol. Avenocoside A and B were also qualitatively identified by TLC [20].

\subsection{Shampoo Formulations}

Different amount of shampoo ingredients including anionic, amphotheric and non-ionic surfactants, preservatives, thickening agent and water were mixed to make a stable shampoo. Fourteen different shampoo formulations were prepared and evaluated for stability and physicochemical tests. Oil extract or total extract with concentration of 2 to $5 \%$ were added to the best shampoo formula. Table 1 shows the composition of a basic shampoo.

Table 1. The composition of a basic shampoo

\begin{tabular}{ll}
\hline Ingredients & Percentages \\
\hline Sodium laureth sulfate & $8-14$ \\
Sodium laureth sulfosuccinate & $1-7$ \\
Cocamidopropyl betaine & $1-5$ \\
Cocamide DEA & $2-4$ \\
Preservative & 0.2 \\
EDTA & 0.1 \\
Deionized water & q.s 100 \\
Total extract/(oil extract) & $2-5 /(0.5-1.5)$ \\
\hline
\end{tabular}

\subsection{Assay of Oat Extract/Oil in the Finished Product}

Ten $\mathrm{ml}$ of $70 \%$ ethanol was added to $2 \mathrm{~g}$ shampoo in a volumetric flask and refluxed for $10 \mathrm{~min}$. After filtration and solvent evaporation, TLC method was used to detect avenocoside A and B in shampoo. Silica gel pre-coated TLC plates were used with a mobile phase of chloroform, glacial acetic acid, methanol and water (64:32:12:8) and anisaldehyde-sulphuric acid reagent.

\subsection{Physicochemical Tests of Shampoos}

Organoleptic assays and physicochemical tests such as $\mathrm{pH}$, viscosity, foam volume (Ross-miles method [21], centrifugation test, stability analysis, and measurement of shampooing activity were conducted as previously described [22].

\subsection{Evaluation of Detergency and Sebum Removal (In Vitro)}

Shampoo detergency was evaluated according to the Thompson's method. Artificial sebum was prepared with this proportion: olive oil $20 \%$, coconut oil $15 \%$, stearic acid 
$15 \%$, oleic acid $15 \%$, paraffin wax $15 \%$ and cholesterol $20 \%$. Hair tresses were cut into 10 inches, $3 \mathrm{~g}$ swatches. The swatches were suspended in $20 \mathrm{ml}$ of $10 \%$ artificial sebum (solubilized in hexane) for 15 min with shaking. Each swatch was then split into two equal samples with $1.5 \mathrm{~g}$ weight. One of the samples was used for shampoo treatment and the other acted as an internal control. The control swatch was left untreated. The sebum remaining in the test and control swatches after shampooing was extracted using $20 \mathrm{ml}$ of hexane. Percentage of sebum removal after shampooing was calculated [23].

\subsection{Clinical Evaluation of Shampoo Efficacy (In Vivo)}

Double -blinded, randomized, placebo controlled, dermatologist supervised clinical studies of shampoo formulations were conducted to determine their effectiveness in the treatment of scalp greasiness and seborrhea. Sixty male and female volunteers age 19 to 30 years old were randomly selected to apply either oat shampoos (extract or oil) or the vehicle shampoo to their scalps for 5 minutes, twice per week for four weeks. The volunteers did not have any previous scalp/hair conditions or on any scalp topical treatment. They had to use just our topical preparation during the study. Signs and symptoms such as scaling, greasiness and itching were assessed every week. Grading of greasiness was done using a 5-point rating scale:

$$
0=\text { none, } 1 \text { =slight, } 2=\text { mild, } 3=\text { moderate, } 4=\text { severe }
$$

The scaling was scored on a 0 to 2 scale (i.e., $0=$ no flakes, $1=$ small flakes, $2=$ large flakes).

The severity of itchiness was scored as: $0=$ no itching, $1=$ moderate itching, $2=$ severe itching [24].Table 2 summarizes demographic features of the test groups before treatment.

Table 2. Demographic features of the tests and placebo groups

\begin{tabular}{llll}
\hline Demographic features & $\begin{array}{l}\text { Shampoo } \\
(\mathbf{O})\end{array}$ & $\begin{array}{l}\text { Shampoo } \\
(\mathrm{E})\end{array}$ & $\begin{array}{l}\text { Shampoo } \\
(\mathrm{V})\end{array}$ \\
\hline $\begin{array}{l}\text { Age }(\mathrm{y}), \text { Mean } \pm S D \\
\text { Gender }(\mathrm{n})\end{array}$ & $24 \pm 2.22$ & $23 \pm 3.1$ & $26 \pm 4.5$ \\
Male & 10 & 12 & 9 \\
Female & 10 & 8 & 11 \\
Scaliness, Mean \pm SD & $1.2 \pm 0.52$ & $1.25 \pm 0.55$ & $1.4 \pm 0.6$ \\
Itchiness, Mean $\pm S D$ & $1.2 \pm 0.7$ & $1.4 \pm 0.5$ & $1.1 \pm 0.72$ \\
Greasiness, Mean $\pm S D$ & $2.05 \pm 1.28$ & $3.1 \pm 1.3$ & $2.35 \pm 1.56$ \\
\hline \multicolumn{3}{l}{}
\end{tabular}

\subsection{Statistics}

To statistically compare subgroups the non-parametric Friedman and Dunn's multiple comparison tests were performed. Kruskal-Wallis test was also conducted to 
compare the groups. A P value $<.05$ was considered significant. The Sigmastat for Windows version 3.5 (Systat software Inc. 2006) was also used.

\section{RESULTS AND DISCUSSION}

Extraction of $100 \mathrm{~g}$ oat flour by using a hydroalcoholic solvent obtained about $4 \mathrm{~g}$ total extract. Hexane was used as a non-polar solvent for extraction of oil from oat flour. About $8 \mathrm{~g}$ oil was obtained from $100 \mathrm{~g}$ oat flour. The Table 3 shows the tests results on oil and extract.

Table 3. Specification of oil and extract

\begin{tabular}{lll}
\hline Specification & Total extract & Oil extract \\
\hline $\mathrm{pH}$ & $4-5.1$ & \\
Specific gravity & NA & 0.919 \\
Refraction index & NA & 1.4719 \\
Saponification index & NA & 197.75 \\
lodine index & NA & 99.82 \\
Color & Opaque green & Clear brown \\
Clear brown & Opaque green & color \\
\hline
\end{tabular}

\subsection{TLC of Oil and Total Extract}

The cleansing activity of oat is mostly attributed to its saponins. Therefore, it is important to determine saponin content of oat in order to evaluate its detergency effect. The total saponin content of Avena sativa was estimated $0.4 \pm 0.1$ (g/kg dry weight, TLC-densitometry) which comply previously recorded estimates. TLC revealed eight to ten gray blue zones in the retention factor $(\mathrm{R} f)$ range of 0.15 to 0.9 . Avenocoside $\mathrm{A}$ and $\mathrm{B}$ are found in the lower $\mathrm{R} f$. The spots had the same $\mathrm{R} f$ of the standard compounds (Fig. 1).

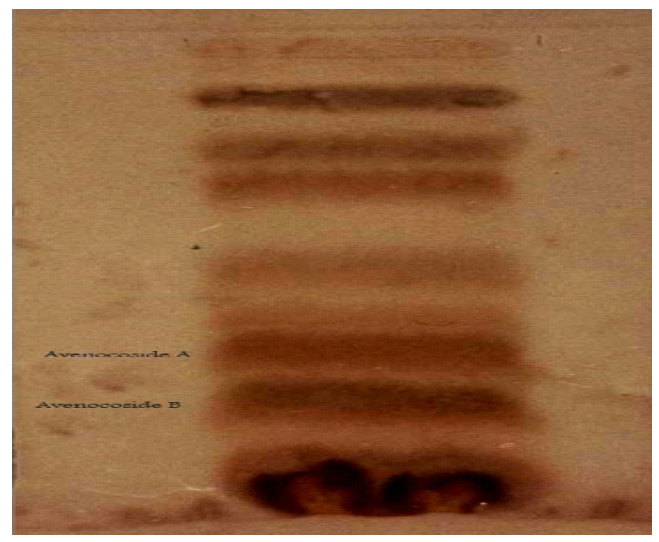

Fig. 1. Thin layer chromatogram of avenocosides A and B. Avenocosides A and $B$ were separated from alcoholic extracts of oat saponins on TLC plates as described in materials and methods 


\subsection{TLC of Shampoos}

TLC method was used to detect presence of Avenocoside $A$ and $B$ in formulated shampoos [25].TLC results revealed that shampoo spots were similar to the oil/extract spots. No spot was detected for the shampoo without oat (Fig. 2). TLC results confirmed the presence of avenocoside $A$ and $B$ in oat shampoos.

\subsection{Physicochemical Tests of Shampoos}

Among fourteen shampoos which formulated and evaluated for stability and physicochemical characterization, the best shampoo was selected. Oil /extract oat with concentration of $2-5 \%$ was added to the best formulation.

\subsection{Detergency Test}

Evaluation of shampoo detergency according to the Thompson's method [23]was done. The range of detergency percentage was $75 \pm 0.5 \%, 78 \pm 0.3 \%$ and $60 \pm 0.2 \%$ for shampoos containing oil, extract and control, respectively. The results indicated a superior detergency power in shampoos containing oat extract and oil compared to that in the control shampoo.

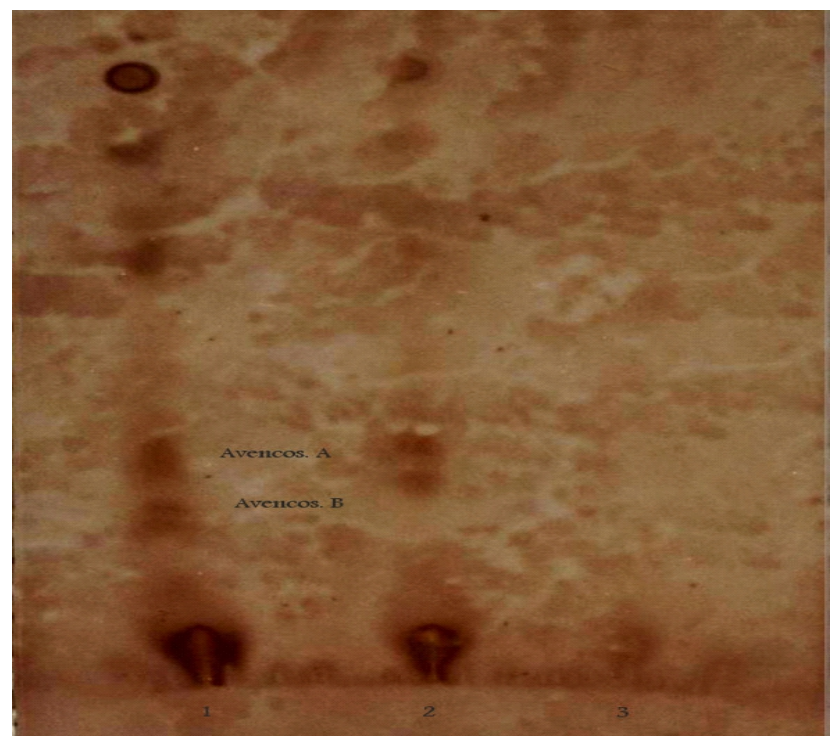

Fig. 2. Thin layer chromatogram of control and oat shampoos. The left spot (1) was from the extract, the middle one (2) was from shampoo containing extract and right spot (3) came from the control shampoo (without extract)

\subsection{Clinical Evaluation of Shampoo Efficacy}

The differences in the medians of itchiness, scaliness and greasiness values among the treatment groups were not statistically significant prior to the experiments. 
Kruskal-Wallis one way analysis of variance on ranks showed that in the subjects treated with shampoos containing oat extract, the difference in the median greasiness values was greater than expected by chance. There was also a statistically significant difference after four weeks treatment $(P=0.003)$. Dunn's method revealed a significant difference between base versus extract and base versus oil, whereas no difference was observed between extract versus oil shampoo $(P<0.05)$.

In terms of itchiness reduction, we did not observe any statistically significant difference among base, oil and extract shampoos. All the tested shampoos failed to reduce scaliness values after four weeks application (Figs. 3 and 4).

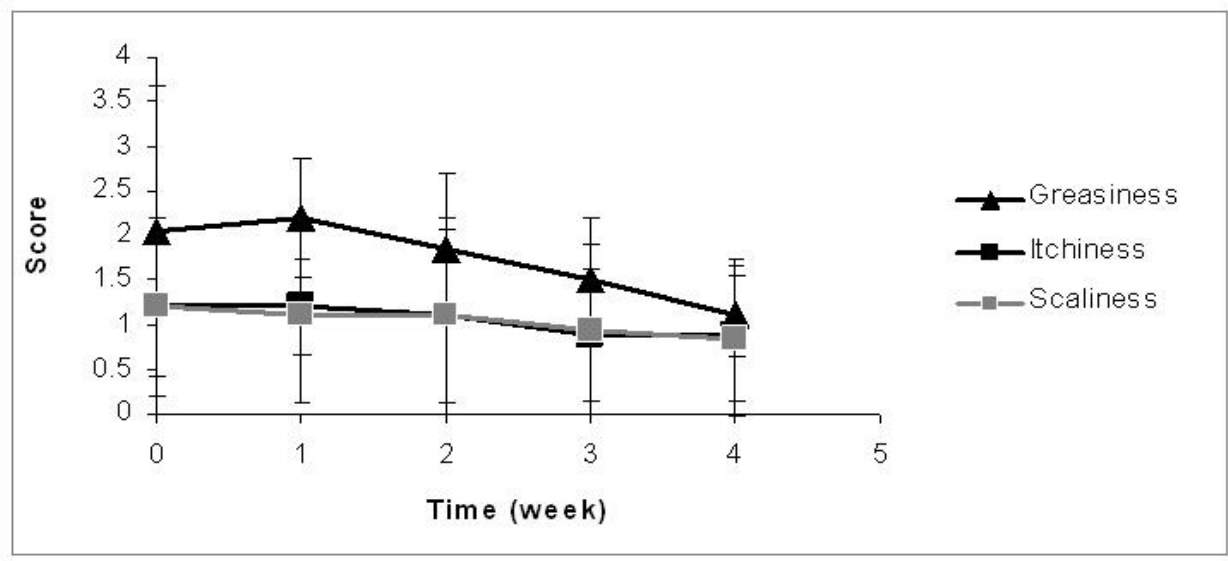

Fig. 3. Reduction in score rating after using the shampoo containing oil of oat. The oat oil containing shampoo was evaluated for reduction of greasiness (black triangle), itchiness (black square), and scaliness (grey square) as described in the material and methods

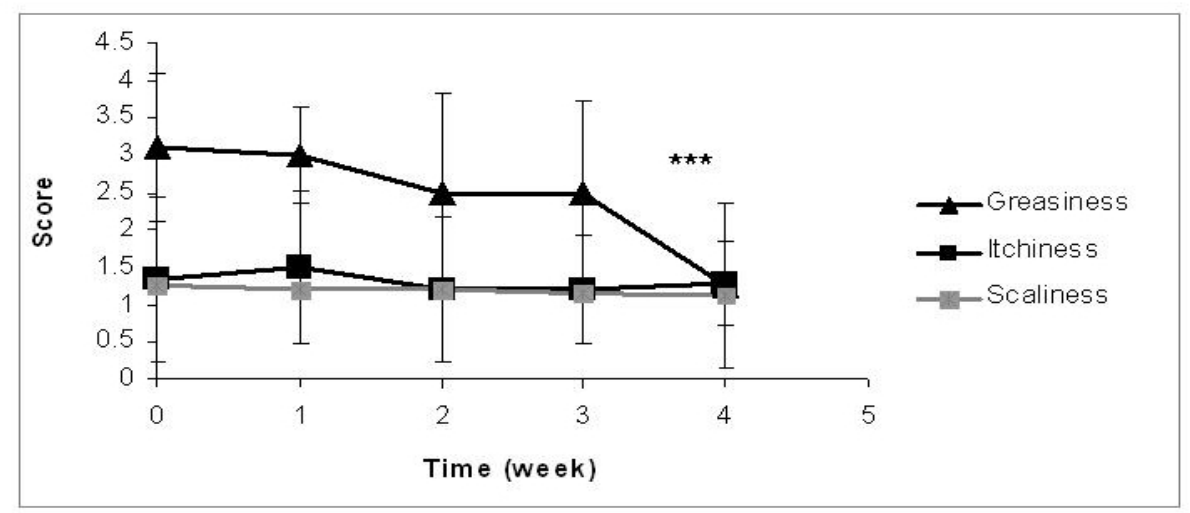

Fig. 4. Reduction of score rating after using the shampoo containing extract of oat. The oat extract shampoo was evaluated for reduction of greasiness (black triangle), itchiness (black square), and scaliness (grey square) as described in the material and methods. ${ }^{* * *}$ Highly significant difference $(P<.005, n=20)$ 
Comparisons of greasiness, scaliness and itchiness scores for base (control) shampoo in the treatment period (four weeks) with the chi-square were not statistically significant (Fig. 5).

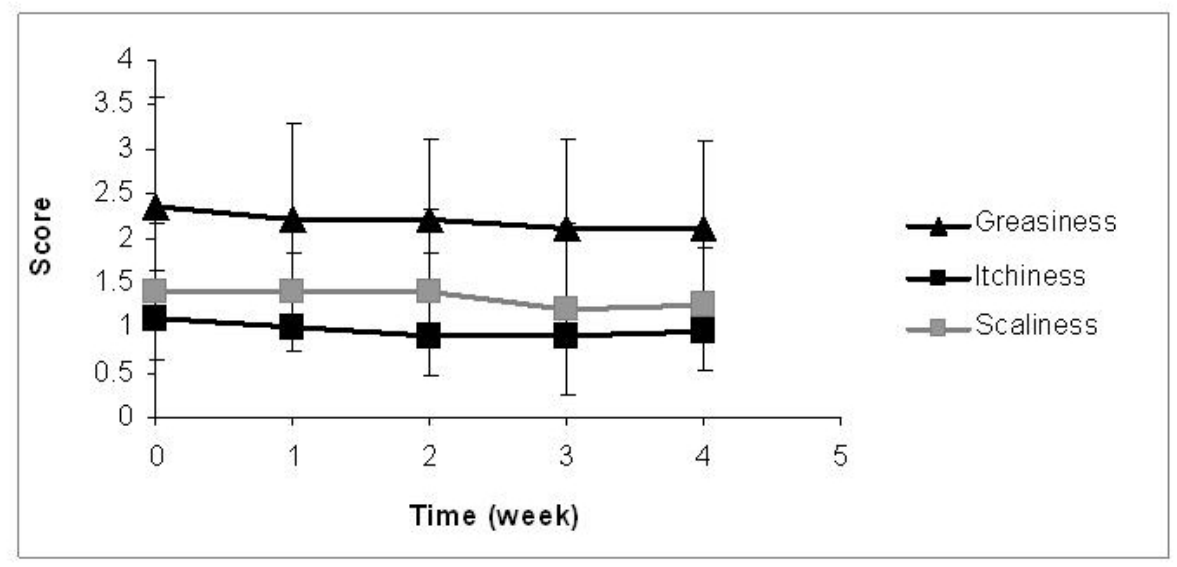

Fig. 5. Reduction of score rating after using the base shampoo. The base shampoo was evaluated for reduction of greasiness (black triangle), itchiness (black square), and scaliness (grey square) as described in material and methods.

The results of the present double blinded study demonstrated that both oat oil and extract shampoos significantly reduced greasiness. Shampoo containing extract appeared to be slightly more effective than shampoo containing oil in reducing greasiness. There was no statistically significant difference for scaliness among all shampoos. Although there are some reports indicating an anti-itching effect for oat $[9,11]$, we did not observe any significant changes in itchiness after using oat shampoos.

Although there are some conflicting reports on adverse reactions of oat products in patients [26-28], in the course of our study we didn't observe any clinical side effects in our subjects; most likely because of short period of contact during shampooing. Our data suggest shampoos containing oat as an appropriate alternative therapy for the management of greasiness in seborrheic patients.

\section{CONCLUSION}

Comparison of Avena sativa extract and oil shampoos with placebo confirmed the potential effect of extract and oil in reducing excretion of sebum from head. Shampoos containing Avena sativa are recommended as a choice product in treatment of greasy hair. 


\section{CONSENT}

All authors declare that written informed consent was obtained from the volunteers and participants in this study.

\section{ETHICAL APPROVAL}

All authors hereby declare that all experiments have been examined and approved by the appropriate ethics committee of the Tehran University of Medical Sciences and have therefore been performed in accordance with the ethical standards laid down in the 1964 Declaration of Helsinki.

\section{ACKNOWLEDGEMENTS}

The authors wish to have an especial acknowledgement for Dr. Khoshayand for his statistical analysis.

\section{COMPETING INTERESTS}

Authors have declared that no competing interests exist.

\section{REFERENCES}

1. Zviak C, Bouillon C. Seborrhoeic conditions (greasy hair). In: Zviak C, ed The science of hair care, pp 98-109. New York: Marcel dekker; 1986.

2. Welch R. The oat crop: production and utilization. London: Chapman and Hall Ltd.; 1995.

3. Collins F. Oat phenolics: avanthramides, novel substituted N-cinnamoy lanthranilate alkaloids from oat groats and hulls. J Agric Food Chem. 1989;37:60-66.

4. Hart J, Polla CH. Oat fractions. Cosmet Toilet. 1998;113:45-52.

5. Martinez-Tome $\mathrm{M}$, et al. Evaluation of antioxidant capacity of cereal brans. $\mathrm{J}$ Agric Food Chem. 2004;52:4690-4699.

6. Sadiq Butt M, et al. Oat: unique among the cereals. Eur J Nutr. 2008;47:68-79.

7. Hauksson JB, et al. Structure of digalactosyldiacylglycerol from oats. Chem Phys Lipids. 1995;78:97-102.

8. Boisnic S, et al. Inhibitory effect of oatmeal extract oligomer on vasoactive intestinal peptide-induced inflammation in surviving human skin. Int $\mathrm{J}$ Tissue React. 2003;25:41-46.

9. Kurtz ES, Wallo W. Colloidal oatmeal: history, chemistry and clinical properties. J Drugs Dermatol. 2007;6:167-170.

10. Saeed SA BN, et al. Inhibitors of prostaglandin biosynthesis in extracts of oat (Aveena sativa) seeds. Biochem Soc Trans. 1981;9:444.

11. Sur R, Nigam A, et al. Avenanthramides, polyphenols from oats, exhibit anti-inflammatory and anti-itch activity. Arch Dermatol Res. 2008;300:569-574. 
12. Baumann $\mathrm{L}$, et al. "Natural" ingredients in cosmetic dermatology. J Drugs Dermatol. 2009;8:s5-9.

13. Cerio R, et al. Mechanism of action and clinical benefits of colloidal oatmeal for dermatologic practice. J Drugs Dermatol. 2010;9:1116-1120.

14. Vie $\mathrm{K}$, et al. Modulating effects of oatmeal extracts in the sodium lauryl sulfateskin irritancy model. Skin Pharmacol Appl Skin Physiol. 2002;15:120-124

15. Matheson JD, et al. The reduction of itch during burn wound healing. J Burn Care Rehabil. 2001;22:76-81; discussion 75.

16. Wu J. Treatment of rosacea with herbal ingredients. J Drugs Dermatol. 2006;5:29-32.

17. Boisnic S, et al. Healing effect of a spray containing Rhealba oat colloidal extract in an in vitro reconstitution model of skin. Int $\mathrm{J}$ Tissue React. 2005;27:83-89.

18. European Medicines Agency. Evaluation of Medicines for Human Use, Assessment report on Avena Sativa L herba and Avena Sativa fructus. Doc. Ref. EMEA/HMPC/202967/2007

19. Webster F. Oat utilization, past, present and future. In: Webster F, ed. Oat: chemistry and technology pp 413-426. St Paul, Minn: American association of cereal chemists Inc; 1986.

20. Wagner H BS, Ricks V. Plantdruganalysis. In: Wagner H BS, Ricks V, ed .A Thin Layer Chromatography Atlas, 2 Edition, p 307. Berlin: Springer-verlag; 2003

21. Ross J MG. An apparatus for comparison of foaming properties of soaps and detergents. Oil Soap. 1941;18:99-102.

22. Trueb RM. Shampoos: ingredients, efficacy and adverse effects. J Dtsch Dermatol Ges. 2007;5:356-365.

23. Thompson D LC, Allen R, Whittam J. Evaluation of relative shampoo detergency. J Soc Cosmet Chem. 1985;36:271-286.

24. Andrew C, et al. Treatment of dandruff with $5 \%$ tea tree oil shampoo. J Am Acad Dermatol. 2002;47:852-5.

25. Murakami S, et al. Prolamellar body and saponins: Avenacosides are not constituents of Avena etioplasts. Plant Cell Reports. 1983;2:148-151.

26. Hardman CM,et al. Absence of toxicity of oats in patients with dermatitis herpetiformis. N Engl J Med. 1997;337:1884-1887.

27. Codreanu F, et al. Risk of allergy to food proteins in topical medicinal agents and cosmetics. Eur Ann Allergy Clin Immunol. 2006;38:126-130.

28. Boussault $P$, et al. Oat sensitization in children with atopic dermatitis: prevalence, risks and associated factors. Allergy. 2007;62:1251-1256.

(C) 2013 Farboud et al.; This is an Open Access article distributed under the terms of the Creative Commons Attribution License (http://creativecommons.org/licenses/by/3.0), which permits unrestricted use, distribution, and reproduction in any medium, provided the original work is properly cited.

Peer-review history:

The peer review history for this paper can be accessed here: http://www.sciencedomain.org/review-history.php?iid=177\&id=12\&aid=929 